\title{
STEM CELLS-BASICS AND PROSPECTIVES
}

\author{
Anushka Banerjee \\ School of Biotechnology \\ KIIT University, Bhubaneshwar, Odisha, India
}

\author{
Arijit Bhattacharya \\ School of Biotechnology \\ KIIT University, Bhubaneshwar, Odisha, India
}

\author{
Neelarghya Ghatak \\ School of Biotechnology \\ KIIT University, Bhubaneshwar, Odisha, India
}

\begin{abstract}
After facing many difficulties scientists in this $21^{\text {st }}$ century have finally been able to use these cells for treating diseases of various types making stem cells a household name in the field of medical sciences. Though the prospective of stem cells began with treating cancer and focusing on it's research, now it's application has spread to various topics like organ regeneration, bone marrow transplant, leukaemia, stroke treatment among others. This paper is a review focused on the discovery of different types of stem cells and the potential aspects based on these cells. The review is summarized by challenges that stem cell therapy must overcome to be accepted in all medical facilities. A wide variety of possibilities makes this cutting edge therapy a turning point in modern medicine, providing hope for untreatable diseases.
\end{abstract}

Keywords - Stem cell, Stroke recovery, Transplantation, Leukaemia, Treatment, Pluripotency, Neural stem cell, Regeneration, Hematopoietic Stem cells, Preleukemic burden

\section{INTRODUCTION}

A. What are stem cells?

A stem cell is a non-specialized, generic cell which can make exact copies of itself indefinitely and can differentiate and produce specialized cells for the various tissues of the body. They are characterized by selfrenewal and potency i.e. - the ability to renew themselves through mitotic cell division and differentiating into a diverse range of specialized cell types. The ultimate stem cell, the fertilized egg, divides five or six times to give rise to branches of cells that form various differentiated organs. During normal tissue renewal in adult organs, tissue stem cells give rise to progeny that differentiate into mature functioning cells. It must be unspecialized, clonogenic and capable of unlimited self-renewal, a process during which a stem cell can divide symmetrically and give rise to two daughter stem cells. It is this capacity to self-renew over a prolonged period of time that ensures that stem cell populations last throughout the life of an organism. Further, it must also be able to divide asymmetrically and give rise to one daughter stem cell and the other daughter cell that in response to appropriate signals can differentiate into multiple types of differentiated cells of all three primitive embryonic germ layers (the ectoderm, mesoderm, and endoderm).

\section{$B$. Symmetric cell division:}

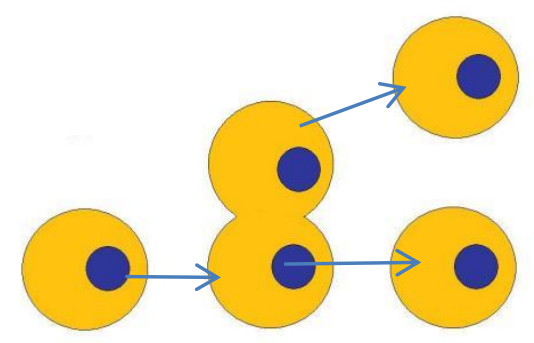

Fig 1: At the beginning of embryonic development, stem cells undergo symmetric cell division. They divide symmetrically, where one cell splits and gives rise to two identical cells that have the same potential. This is why we said the stem cells in the early embryo remain the same and are identical.

\section{Asymmetric cell division:}

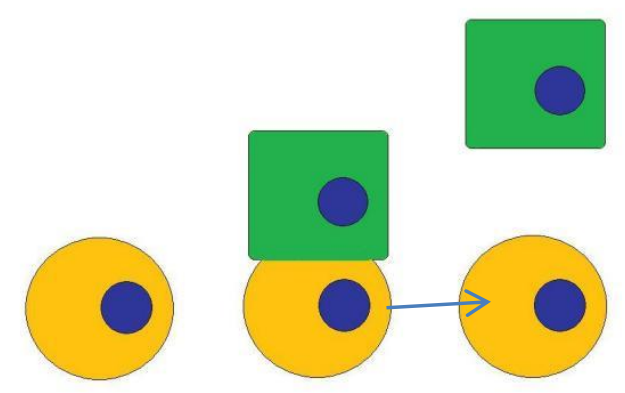

Fig 2: Then at blastocyst formation and gastrulation the stem cells start to divide asymmetrically. When these stem cells divide, they give rise to two cells that are different from each other. One of the cells remains a stem cell-the yellow oneand the other changes into a progenitor cell- the green square. That progenitor cell is a young cell that will change into a mature cell type, like the epithelial cell in green. Asymmetric cell division is when a stem cell divides to produce two cells that are different from each other. 


\section{International Journal of Engineering Applied Sciences and Technology, 2021 Vol. 6, Issue 5, ISSN No. 2455-2143, Pages 178-188 \\ Published Online September 2021 in IJEAST (http://www.ijeast.com)}

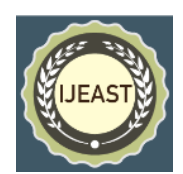

\section{PAST ASPECT OF STEM CELL RESEARCH}

Stem cell was a fascinating topic from a very long time. Scientists got the idea about stem cell research from the regenerative abilities of some animals. In earlier time stem cell was derived from germ cells, embryonic cells (EGCs), fetus and adult. Pluripotent EC cells (Enterochromaffin Cells) were the first kind of stem cells that were recognized in terminally differentiated tissues of spontaneously occurring murine tumours of a mice. They can be differentiated through the process of in vivo as well as in vitro. The EC cells have a very similar characteristics and behaviour to ES cells (Embryonic Stem cells). And this served as a model to isolate comparable cells from mammalian embryos. So the Embryonic stem cells were first derived from some certain strains of mice.

The research with stem cell faced a lot of ethical and debating issues from the very beginning and generated a great deal of public interest. When the field started advancing with it's research and perspectives it raised as many new questions as it has answered. Scientists took up the responsibility to dispel misconceptions about Embryonic Stem cell research. The myths raised that Human Embryonic Stem Cells are derived from aborted fetuses and have the potential to form the whole human being, took a lot of effort and time to dismissed. Scientists started their research with a vision to develop cellreplacement therapies with the application of regenerative medicine and later-on that opened up a lot more application and prospective of stem cell on cell-based therapies.

The most tissue repair process in mammals are resulting from the activation of pre-existing stem cells. This pre-existing stem cell is known as progenitor cells. The general type of stem cells can replicate indefinite times but progenitor stem cells can not differentiate indefinitely, they can only form multiple types of specialized cells. Stem cells or progenitor cells are the common denominator for nearly all types of regeneration or cell repairing process. Either they can be pre-existing, as is the case for mammals, or can be created by the process of dedifferentiation. The historical success of bone marrow transplants have opened the door for various treatment and therapies that have improved the lives of many patients suffering from leukaemia, blood disorders and other immunological disorders. Clearly, stem cell research leading to prospective therapies in reparative medicine has the potential to affect the lives of millions of people around the world for the better good reason

\section{SOURCES OF STEM CELLS}

\section{A. Classification of stem cells on the basis of potency :}

Stem cells can be classified by the extent to which they can differentiate into different cell types. These four main classifications are totipotent, pluripotent, multipotent, or unipotent.
Totipotent stem cells -have the potential to become any kind of cell in the body. After an egg is fertilized, it undergoes a series of divisions to become an embryo and later a fetus. The cells that are formed during these first few divisions are totipotent, i.e., each cell can form a complete organism. Embryonic stem (ES) cells are taken from inside the blastocyst, a very early stage embryo. The blastocyst is a ball of about 50-100 cells and it is not yet implanted in the womb. It is made up of an outer layer of cells, a fluid-filled space and a group of cells called the inner cell mass. ES cells are found in the inner cell mass.

Pluripotent stem cells result after totipotent stem cells undergo the first few divisions. Pluripotent stem cells include cells from the blastocyst stage of the embryo. Given the right signals, a pluripotent stem cell could turn into any cell in an organism (except placenta), potentially growing into tissue for a heart, a kidney or bone.

Multipotent cells can be isolated from many tissues of the body and function as a repair system for damaged tissue. As compared to the totipotent and pluripotent stem cells, they possess a limited ability to differentiate into other cell types. Adult stem cells from the blood, nervous system and heart represent multipotent stem cells.

Oligopotent- The ability to differentiate into a few cells. Examples include (adult) lymphoid or myeloid stem cells.

Unipotent -The ability to only produce cells of their own type, but have the property of selfrenewal required to be labeled a stem cell. Examples include (adult) muscle stem cells.

\section{$B$. Classification of stem cells on the basis of their sources:}

Embryonic stem cells- come from a five to six-day-old embryo. They have the ability to form virtually any type of cell found in the human body

Embryonic germ cell-are derived from the part of a human embryo or fetus that will ultimately produce eggs or sperm (gametes)

Tissue/adult stem cells-are undifferentiated cells found among specialized or differentiated cells in a tissue or organ after birth. Based on current research they appear to have a more restricted ability to produce different cell types and to self-renew.

Induced pluripotent stem cells-area type of pleuripotent stem cell artificially derived from a non-pluripotent cell, typically an adult somatic cell, by inducing a 'forced" expression genes.

\section{STEM CELL NICHES AND LINES}

Microenvironment around stem cells that provides support and signals regulating self-renewal and differentiation.

The niche can act on a stem cell by various mechanisms:

- Direct contact between the stem cell and the niche cells

- Soluble factors released by the niche that travel to the stem cell 


\section{International Journal of Engineering Applied Sciences and Technology, 2021 Vol. 6, Issue 5, ISSN No. 2455-2143, Pages 178-188 \\ Published Online September 2021 in IJEAST (http://www.ijeast.com)}

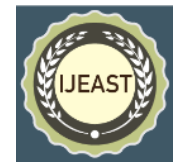

- Intermediate cells that 'communicate' between the niche and the stem cell

A stem cell line is a family of constantly dividing cells, the product of a single parent group of stem cells. They are obtained from human or animal tissues and can replicate for long periods of time in vitro. They are frequently used for research relating to embryonic stem cells or cloning entire organism . Once stem cells have been allowed to divide and propagate in a controlled culture, the collection of healthy, dividing, and undifferentiated cells is called a stem cell line.

\section{MESENCHYMAL STEM CELLS}

Mesenchymal stem cells(MSCs) are multipotent stem cells found in bone marrow that are important for making and repairing skeletal tissues such as cartillage, bone and the fat found in bone marrow.MSCs treatments are being developed to help repair bone and cartillage such as injuries to the knee meniscus or long term accumulated damage that leads to osteoarthritis.MSCs were originally found in the bone marrow. There have since been many claims that they also exist in a wide variety of other tissues, such as umbilical cord blood, adipose (fat) tissue and muscle. It has not yet been established whether the cells taken from these other tissues are really the same as, or similar to, the mesenchymal stem cells of the bone marrow.

The bone marrow contains many different types of cells. Among them are blood stem cells(also called hematopoietic stem cells; HSCs) and a variety of different types of cells belonging to a group called 'mesenchymal' cells. Only about $0.001-0.01 \%$ of the cells in the bone marrow are mesenchymal stem cells.

The MSCs in bone marrow can arrive by three different mechanisms:

(1) They can enter along with the vasculature;

(2) they can migrate into the space after vascularization along the vessel paths, i.e., from the periosteum, which has documented multipotentiality; or

(3) they can arrive via the blood proper, indicating the existence of a circulating MSCs.

The first two mechanisms differ only with respect to the timing of MSC entrance into the marrow, not by the path of arrival or migration. The third mechanism differs from the other two only with respect to which side of the capillary basement membrane the MSCs use, the luminal or abluminal. However, this mechanism of MSC migration or circulation has important implications for adult tissue repair in that it may be possible to deliver reparative MSCs via the circulation as opposed to only localized applications, especially if MSC docking sites exist on the endothelium lining the vascular network.The first and second mechanisms of MSC arrival (along with the vasculature) to the bone marrow space are supported by two distinct observations and experiments with the vascular-associated cells (pericytes), and on smooth muscle cells from marrow.

MSCs within marrow also function as a reservoir of stem cells for the repair of bone fractures and for the natural turnover of bone. The sequence of cellular events in fracture repair includes the formation of a clot, migration of macrophages and "mesenchymal" cells into the site, formation of cartilage in the fracture callus, and the bridging of the fracture via endochondral ossification. The MSCs are the cells that form both the cartilage callus and the bone that bridges the repair site. MSCs also play an osteogenic role in normal bone remodeling or turnover. During natural bone turnover, hematopoietic lineagederived osteoclasts resorb portions of bone in "resorption pits," the osteoclasts expire, and the excavated pits are then refilled with new bone by osteoblasts that differentiate from MSCs. The MSCs are the reservoir for osteoblastic cells that are needed for the turnover process that occurs throughout an organism's lifetime. It is hypothesized that diminished numbers of MSCs or the restricted access to MSC reservoirs is responsible for some agerelated bone loss.

While it has generally been accepted that cells retained some plasticity (the ability to transdifferentiate or dedifferentiate and redifferentiate into other cell types), especially in the developing embryo, recent results on the differentiation of some mesenchymal phenotypes suggest that plasticity may be more common than previously thought. Mesenchymal tissues may be particularly prone to phenotype plasticity than the other germinal layers, although recent results suggest that even neural tissues may exhibit plasticity toward the hematopoietic lineage and hematopoietic stem cells may differentiate into hepatic oval cells. Another characteristic of mesenchymal cells from marrow is that they often have overlapping functions and phenotypes. The ability of MSCs to be expanded in culture, coupled with their potential to differentiate into end-stage mesenchymal phenotypes, makes MSCs a likely source of cells for tissue repair strategies. One advantage of the use of MSCs is that cells are easily obtained by a standard, outpatient bone marrow harvest. The cells can then be expanded and used for autologous transplantation. The key obstacles to overcome are expansion while retaining differentiation potential, delivery of cells to the site of repair, promotion of differentiation toward the appropriate mesenchymal phenotype, and the integration of the differentiated tissue into the host repair site. Studies have demonstrated the feasibility of using MSCs to augment the repair of bone and to augment the integration of scaffold materials into bone sites. MSCs have also been tested in tendon repair models, which showed that MSCs coimplanted in a collagen matrix carrier were able to integrate into the neotissue and that the implanted MSCs became aligned in parallel with the fibers and native cells.MSCs are chondrogenic, so another tissue engineering application is for the repair of cartilage, a tissue that is notorious for its 


\section{International Journal of Engineering Applied Sciences and Technology, 2021 \\ Vol. 6, Issue 5, ISSN No. 2455-2143, Pages 178-188 \\ Published Online September 2021 in IJEAST (http://www.ijeast.com)}

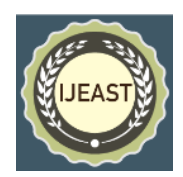

inability to heal, especially in adult animals. A number of studies have been conducted on the repair of articular cartilage defects that have been implanted with MSCs contained within different polymers, such as hyluronic acidbased polymers, type I collagen, and polylactic acid. A major obstacle to the application of MSCs in cartilage repair is improving the integration of neocartilage matrix with the surrounding native cartilage matrix. MSCs have been tested as therapeutic agents for the repair of brain injury. MSCs have also been shown to promote peripheral nerve repair. Another potential application for MSCs is the systemic introduction of osteogenic cells to counteract age-related osteoporosis or as a potential cure for osteogenesis imperfecta . To effectively treat these diseases, it is necessary to devise a method for the systemic introduction of MSCs.

The attractiveness of MSCs as targets for gene therapy lies in three primary considerations:

(1) MSCs are easy to obtain by bone marrow aspiration, with little or no morbidity to the patient;

(2) MSCs are a homogeneous population of cells that can be easily expanded; and

(3) MSCs have the potential to differentiate into multiple cell phenotypes, which may make them useful for targeting specific tissues.

Applications for MSCs to correct a variety of disorders in the musculoskeletal system and for systemic delivery of genetically engineered products is still in the early stages of development. The use of MSCs for bone repair is probably the most advanced with respect to potential for clinical applications.

\section{HEMATOPOIETIC STEM CELL}

Alexander Maximow, working in St. Petersburg as a military doctor in 1909, was the first to suggest that there is a hematopoietic stem cell with the morphological appearance of a 'lymphocyte' capable of migrating through the blood to microecological niches that would allow them to proliferate and differentiate along lineage specific pathways. HSC are nowadays defined as clonogenic cells that can self-renew and maintain multilineage hematopoiesis. Multipotent HSC differentiate into oligolineage progenitor cells which lose their self-renewal capacity and themselves give rise to mature blood cells. The generation of mature blood cells from pluripotent HSC involves a highly regulated progression through successive stages as commitment to a specific cell lineage, terminal differentiation of lineage-restricted progenitor cells, growth arrest and apoptosis. The expression of different receptors on the surface of hematopoietic progenitors permits the interaction with various regulatory elements present in their environment, which includes stromal cells, extracellular matrix molecules and soluble regulatory cytokines as growth and differentiation factors.

\section{A. The Formation of the Hematopoietic System}

THE EARLY EMBRYO Hematopoiesis begins in the mouse on day 7 of gestation in the yolk sac. This event, coupled with the observed migration of cells from the yolk sac to the embryo body, gave rise to the suggestion that the yolk sac is the only site of de novo stem cell formation and that all other hematopoietic tissues are seeded by HSC, which ultimately arise in the yolk sac

ES CELL DIFFERENTIATION ES cells are perpetually selfrenewing, totipotent progenitors derived from the inner cell mass of bIastocysts. ES cell lines can contribute to the formation of all tissues thus far examined, including germ and hematopoietic cells . In culture, ES cells can form embryoid bodies containing endodermal, mesodermal, and ectodermal tissues, with structures that include blood islands and erythroid cells . In vitro, ES cells can differentiate into embryonic and adult erythrocytes, macrophages, granulocytes, and mast cells. The Transition to Adult Hematopoiesis-The fetal liver is the principal site of mid- and late-gestation hematopoiesis in mammals, beginning at 10 days post-conception in mice. The adult hematopoietic system begins to take shape in the fetal liver and includes the emergence of a large and expanding pool of multipotent progenitors.

\section{B. Preleukemic HSCs:}

Acute myeloid leukemia is the cancer of bone marrow and blood, it is an aggressive malignancy which can also arise from the accumulation of mutations within hematopoietic stem cells (HSCs). leukemia-specific mutations have been termed as "pre-leukemic", so this type of HSCs are known as "Preleukemic HSCs" and they are also considered as the ancestors of leukemia. Till now no studies have provided any type evidence about upper and lower bound for the period of time between the acquisition of the first leukemogenic mutation and the onset of disease.

The current model for leukemogenic mutations or preleukemic clonal evolution has resulted from the scientific evidence varying from mouse models to high-throughput sequencing of primary human specimens. Which posits that the first leukemogenic mutation must either occur in a cell that is capable of self-renewal or confer self-renewal upon the cell and If the first mutation fails to meet one of these two criteria, it will be lost over time due to terminal differentiation. This model helped to get the overview of preleukemic HSCs in details and that helped to know a lot more about the effect and importance of hematopoietic stem cells on leukemia.

We define the percent of HSCs in a leukemia patient as "preleukemic burden," this harbor at-least the first preleukemic mutation. In this way, patients whose preleukemic HSCs have expanded greatly will have a high preleukemic burden. the preleukemic burden across AML patients can vary from $100 \%$ to below the limit of detection of standard high-throughput sequencing methodologies $(\sim 1 \%)$. Which means a single HSC expanded to outcompete all other 


\section{International Journal of Engineering Applied Sciences and Technology, 2021 Vol. 6, Issue 5, ISSN No. 2455-2143, Pages 178-188 \\ Published Online September 2021 in IJEAST (http://www.ijeast.com)}

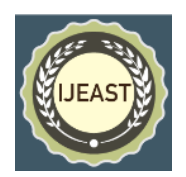

HSCs after acquisition of the first preleukemic mutation can be said that it's preleukemic burden is $100 \%$.

So from all the above information it can be highlighted that the preleukemic HSCs outcompete normal HSCs, they have the ability to survive long and they undergo clonal evolution through the acquisition of multiple additional mutations, eventually leading to frank leukemia. The mutation in those stem cells make it more stronger and increase it's "fitness". A stem cell that produces more daughter cells whose selfrenewal potential is at least as great as the parental cell would have an increased fitness. In case of a preleukemic stem cell, it is not sufficient to merely produce more daughter cells. Rather, those daughter cells must retain the ability to selfrenew if they are to persist long enough to acquire additional preleukemic and eventually leukemic mutations. The mutations enable the HSCs to persist long enough to acquire additional mutations and prevent full differentiation during the leukemic phase of AML.

\section{THE EVOLUTION OF CANCER STEM-CELL RESEARCH}

From the very earlier time bone-marrow reconstitution experiments and other application of stem cell research and therapies have opened the door of cancer stem cell research. At the very beginning of the research On 1855, Rudolph Virchow proposes the 'embryonal-rest hypothesis' of tumour formation, based on histological similarities between tumors and embryonic tissues. This theory, later on expanded by other pathologists such as Julius Cohnheim, and they suggested that tumours develop from residual embryonic tissues. On 1961 James Till and Ernest McCulloch suggested the existence of the haematopoietic stem cells, based on experiments demonstrating the ability of bone marrow to provide protection to lethally irradiated mice. On 1963 Robert Bruce and Hugo Van der Gaag9 demonstrated the low in vivo clonogenic efficiency of mouse lymphoma cells. 1967-1981 was a very crutial period as Philip Fialkow and coworkers use patterns of X-linked gene inactivation in patient samples to demonstrate that leukaemias and myeloproliferative disorders are clonal in origin. These studies also indicate the involvement of an early stem or progenitor cell in chronic myelogenous leukaemia and acute myelogenous leukaemia (AML). Meantime on 1968 fluorescenceactivated was developed for cell sorting procedure by Leonard Herzenberg and coworkers and this research continued till 1973. That time the production of the first commercially available machines also induced the research. On 1970 Van R. Potter, Barry Pierce and other investigators extend the embryonal-rest hypothesis to suggest that tumours arise from maturation arrest in tissue-specific stem cells.

1980 was a remarkable year as Monoclonal antibodies were created against numerous haematopoietic cellsurface antigens, in the same year scientists catheterized severe combined immunodeficiency (SCID) mutation in mice. And at the same time professor Jim Griffin, Bob Lowenberg and co-workers demonstrated the Low clonogenic efficiency of AML blasts in in-vitro procedure. On 1988 a great thing happend in the history of stem cell and cancer research, Professor Irving Weissman and colleagues used SCID mice as an assay system for human haematopoietic cells for the first time. In 1989 the refinement of SCID and non-obese diabetic (NOD)/SCID mouse assays for assessment of normal and leukaemic stemcell function was done by John Dick and colleagues, as well as other investigators and this continued till 1994. After 1990s many improvement happained in the field of stem cell and cancer research, Characterization of the NOD/SCID immunocompromised mouse happened in that time. After 1994 the cell sorting with the commercial manufacture of multiparameter high speed machines advanced a lot.

In 1997 Dr. John Dick and coworkers Identified the first cancer stem cell, the leukaemia stem cell, insamples from patients with AML which made that year very significant. And as the research continues on this field Michael Clarke and colleagues in 2003 described the identification of the breast cancer stem cell - the first stem cell to be identified from a solid tumour. In 2004 Peter Dirks and colleagues defined a cancer stem cell in tumors of the central nervous system, and this opened the chances of application of stem cell research in the field of neuroscience. This further showed a chance of application of stem cell research on the cancer of nervous system and also in case of stroke.

\section{STEM CELL RESEARCH IN STROKE}

Neuronal stem cells are responsible for the rise of entire nervous system during developmental phase. Studies done with animal model shows that stem cell transplantation can improve or restore function after a stroke by replacing neurons, modulation of inflammation, processing remyelination and axonal plasticity. Endogenous neuronal stem cells are a special type of stem cells who are also theraputic targets because they can produce neurons after a spinal cord injury or after a stroke. Researches are going on with this idea but currently there is no proven stem cell-based therapy for stroke. Improvement in a patient with stroke could be achieved through neuronal replacement is a approach taken by the scientists as the study shows stem cells can survive, differentiate into neurons, and partially restore function after transplantation in the stroke-damaged rodent brain. The findings from the rodent model experiment provided evidence that the stem cell-derived neurons can exhibit some level of synaptic integration in the host neural circuits. It also suggested that neuronal replacement in the stroke damaged brain may contribute to behavioral improvements. Human Embryonic Stem cells can give rise to unlimited numbers of 


\section{International Journal of Engineering Applied Sciences and Technology, 2021 \\ Vol. 6, Issue 5, ISSN No. 2455-2143, Pages 178-188 \\ Published Online September 2021 in IJEAST (http://www.ijeast.com)}

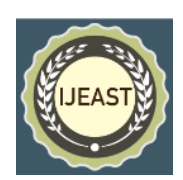

Neuronal Stem Cells, but there is a problem in that approach as they are associated with a risk of tumor formation. Though scientists are working on stem cells programming to prevent the chances of tumor formation through this approach.

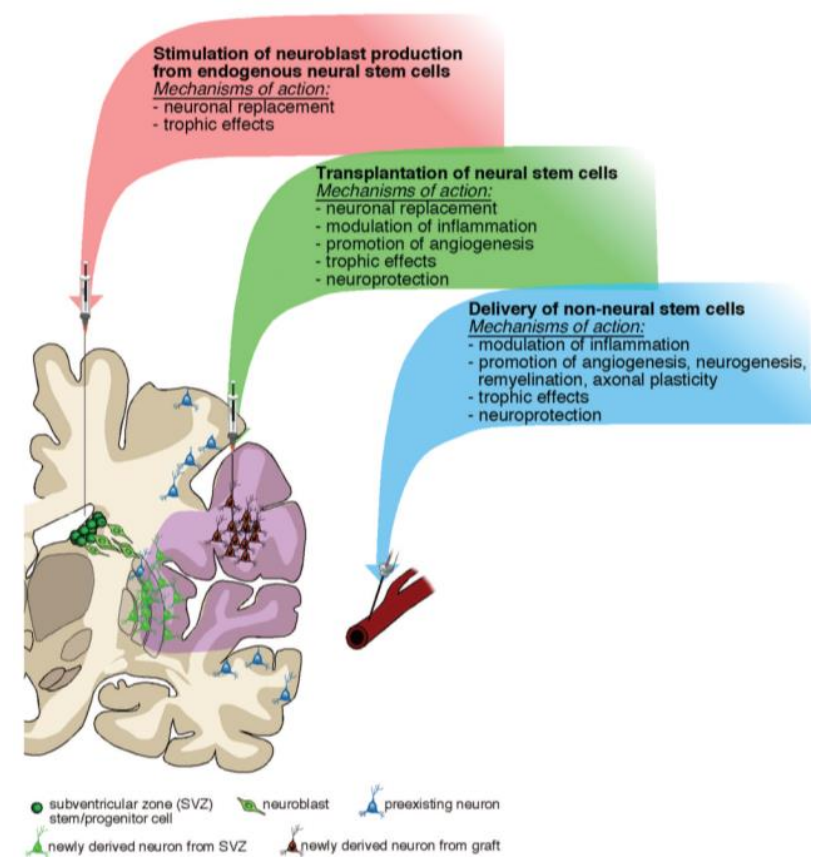

Fig 3: Stem cell-based approaches for stroke therapy.

\section{Area of approaches in Stem Cell and Stroke research:}

Though currently there is no proven stem cell-based therapy for stroke, but the advancement of researches are taking the approach of stem cell in clinical application for stroke patients These advancements in both experimental and clinical research is particularly focusing in the following areas,

- Most stem cells delivered in animal models are a heterogeneous population and it is unclear that which specific cell types are important for functional recovery, so it is important to have a good knowledge about mechanism of that stem cell's action. The cellular and molecular mediators of immune-modulation,, axonal plasticity, and neuroprotection induced by stem cells are needed to be characterized in details in order to avoid any type of complications

- The animal model of stroke experiment cannot reflect the after effects of stroke ( ex: memory-loss, diabetes, atherosclerosis, or hypertension) that occurs in case of humans.So the behavior of stem cells after successful transplantation in animal models may only partially reflect how these cells will behave in case of a human patient. For successful clinical translation, it is necessary to do the experiment in animal models that the stem cellbased approach induces substantial improvement of functional deficits which resemble the debilitating symptoms in patients with stroke.

- Till now the research have find that stem cell can reform neuronal cells after stroke but it is still unknown that which patients with stroke will get the most benefit from this therapy. Clinical trials have provided some information about selection of patients for approaches acting through mechanisms other than the neuronal replacement. Degree of sensorimotor and cognitive impairments caused by the prospects of spontaneous recovery, type of neuron generated from stem cells; localization and extent of the ischemic damage can lead to the selection of patient for future stem cell-based neuronal replacement strategy.

- To access the traces of stem cell, the distribution of the damage, monitor stem cell's survival and their effect on the patient's brain, noninvasive imaging will be crucial way. The existing methods such as MRI, PET-Scan (positron emission tomography) can trace the transplanted stem cells in vivo. A recent study by two scientists Rueger and coworkers showed that PET-Scan can also measure the mobilization of endogenous NSCs (Neuronal stem cell) in the SVZ (Subventricular zone) after stroke. Although several techniques may have to develope in order to get more high resolution imagining on the effect of stem cell in stroke patients.

Focusing on these areas will surely provide supportive evidence that stem cell-based approaches can be developed into clinically useful strategies to promote recovery after stroke

\section{IPS STEM CELL TECHNOLOGY}

Induced pluripotent stem (iPS) cells, are a type of pluripotent stem cell derived from adult somatic cells that have been genetically reprogrammed to an embryonic stem (ES) cell-like state through the forced expression of genes and factors important for maintaining the defining properties of ES cells. The breakthrough discovery of iPS cells allow researchers to obtain pluripotent stem cells without the controversial use of embryos, providing a novel and powerful method to "dedifferentiate" cells whose developmental fates had been traditionally assumed to be determined. Furthermore, tissues derived from iPS cells will be a nearly identical match to the cell donor, which is an important factor in research of disease modeling and drug screening. iPSC are derived from skin or blood cells that have been reprogrammed back into an embryonic-like pluripotent state that enables the development of an unlimited source of any type of human cell needed for therapeutic purposes. For example, iPSC can be prodded into 


\section{International Journal of Engineering Applied Sciences and Technology, 2021 \\ Vol. 6, Issue 5, ISSN No. 2455-2143, Pages 178-188 \\ Published Online September 2021 in IJEAST (http://www.ijeast.com)}

becoming beta islet cells to treat diabetes, blood cells to create new blood free of cancer cells for a leukemia patient, or neurons to treat neurological disorders.

Human iPSCs are a promising prospect for cell therapy in a wide range of diseases for which there are currently no cures or effective therapies, such as neurodegenerative diseases of the central nervous system, heart infarction, diabetes mellitus, and diseases of the liver, lung, and kidney. Given that iPSCs can be produced in a patient-specific manner, they may be used in autologous transplantation-avoiding complications of rejection by the host immune system. Various methods have been adopted to induce pluripotency in somatic cells which can be categorized into integrative approaches, in which foreign DNA sequences encoding reprogramming factors are inserted into the genome of the starting cells, and nonintegrative methods, which do not require permanent genetic modification. Although iPSCs generated using integrative methods can be effectively used to conduct basic studies, discover new drugs, screen toxins, and model diseases in vitro, non-integrative methods offer the significant advantage to potentially produce "safe" iPSCs in which the potential of acquiring secondary disease-causing mutations is minimized and are thus considered to be more suitable for cell-based therapies.

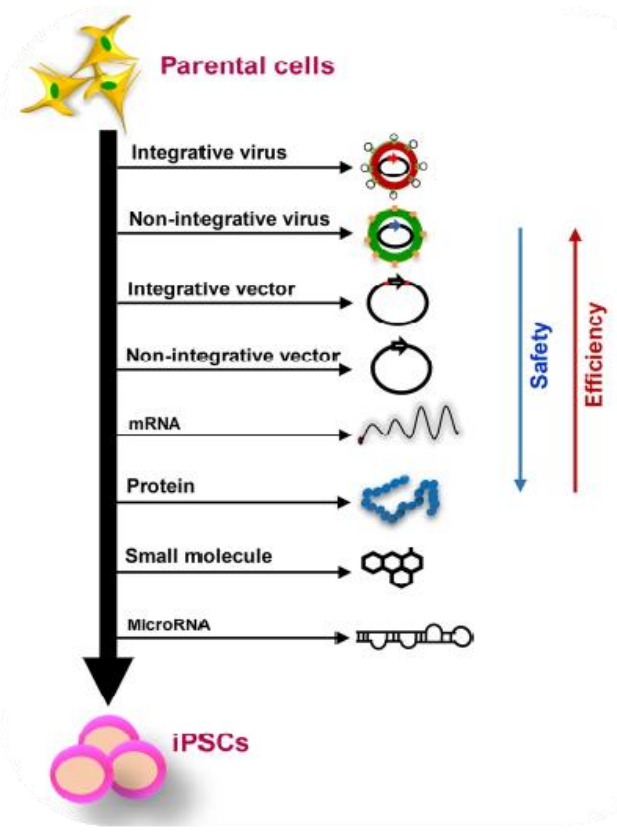

Fig 4: Various methods and approaches are used to convert somatic cells into iPSCs. Integrative methods such as integrative viruses and vectors provide the highest reprogramming efficiency but the lowest safety. In contrast, non-integrative approaches such as the use of small molecules and microRNAs tend to have a less reprogramming efficiency. Notably, episomal vectors, which do not integrate with the host cell's genome, appear to provide both a high efficiency of
iPSC generation and sufficient degree of safety. Although all the illustrated approaches could potentially be used to produce iPSCs for applications such as basic research, drug screening, and disease modeling, genomic integration should be avoided for generation of clinical-grade iPSCs.

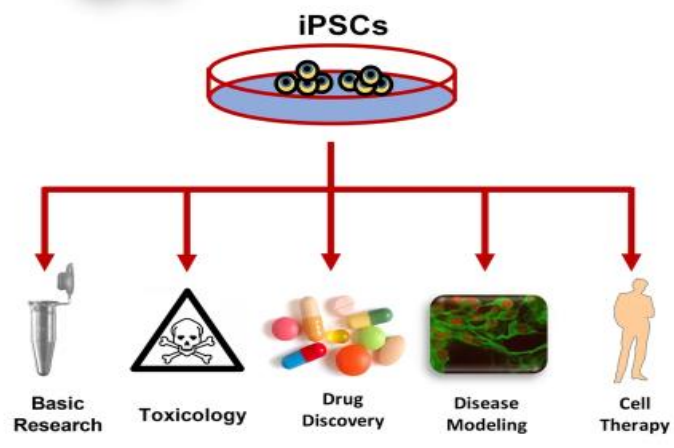

Fig 5: Because of immortality and immense differentiation potential, iPSCs have all the potential biomedical applications of ESCs. They can be used to model pluripotency and multilineage differentiation in vitro, screen and discover new drugs, and establish disease models in a dish. iPSCs also hold a great potential to be used for replacing diseased or lost tissues, which needs specific considerations to provide safe, clinicalgrade cells for transplantation into patients.

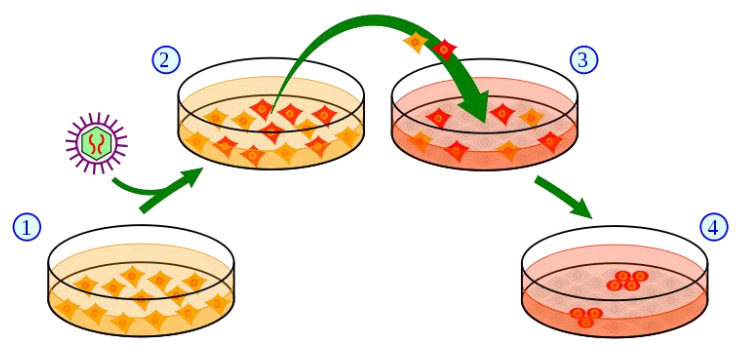

Fig 6: Creating induced Pluripotent Stem cells is like turning back the clock in a subset of cells. This method creates pluripotency, rather than directly harvesting or cloning embryonic stem cells.

In induced Pluripotent Stem Cell technology, you FIRST isolate and culture skin cells from a patient. SECOND, you introduce three or four pluripotency genes into the skin cells by using an engineered virus carrier. The expression of these genes regenerates the stem cell phenotype.The viruses simply deliver the genes of interest and are themselves engineered not to be harmful.

Here, the red cells indicate the cells actively expressing the four essential, stem cell or pluripotency, genes.

In the THIRD image, after the skin cells have been genetically engineered, you harvest and culture them according to the method for embryonic stem cell culture - that is, together with 


\section{International Journal of Engineering Applied Sciences and Technology, 2021 Vol. 6, Issue 5, ISSN No. 2455-2143, Pages 178-188 \\ Published Online September 2021 in IJEAST (http://www.ijeast.com)}

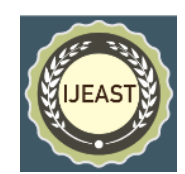

feeder cells. FINALLY, through this process, a subset of the cells generates embryonic-stem-cell-like colonies called induced Pluripotent Stem cells.

\section{Pros and Cons to iPS cell technology- \\ Pros:}

Cells would be genetically identical to patient or donor of skin cells (no immune rejection!)

Do not need to use an embryo to get pluripotent stem cells

Cons:

Cells would still have genetic defects

One of the pluripotency genes is a cancer gene

Viruses might insert genes in places we don't want them (causing mutations)

\section{FUTURE OF STEM CELL}

In recent years, stem cell therapy has become a very promising and advanced scientific research topic. The development of treatment methods has evoked great expectations and have offered much hope by promising to greatly extend the numbers and range of patients who could benefit from transplants, and to provide cell replacement therapy to treat debilitating diseases such as diabetes, Parkinson's, Alzheimer's and Huntington's disease.

Stem cell technologies have applications for basic science (study of complex processes), medical research (to produce large numbers of genetically uniform cultures of organ tissuesfor example, liver, muscle, or neural), and therapies (repair or replace damaged or diseased tissues). Human stem cell research holds enormous potential for contributing to our understanding of fundamental human biology. With the use of tissue engineering, various scientific discoveries in the past two decades have led to the identification of the limbal location of corneal epithelial stem cells and the role it plays in regenerating the corneal epithelium.

Stem cells have great potential to become one of the most important aspects of medicine. Stem cells can be used in new drug tests. Each experiment on living tissue can be performed safely on specific differentiated cells from pluripotent cells to test the drugs properly, the conditions must be equal when comparing the effects of two drugs. To achieve this goal, researchers need to gain full control of the differentiation process to generate pure populations of differentiated cells.

Multipotent haematopoietic stem cell (HSC) transplantation is currently the most popular stem cell therapy. Target cells are usually derived from the bone marrow, peripheral blood, or umbilical cord blood. The procedure can be autologous (when the patient's own cells are used), allogenic (when the stem cell comes from a donor), or syngenic (from an identical twin). HSCs are responsible for the generation of all functional haematopoietic lineages in blood, including erythrocytes, leukocytes, and platelets. HSC transplantation solves problems that are caused by inappropriate functioning of the haematopoietic system, which includes diseases such as leukaemia and anaemia.

It is possible not only to delay the progression of incurable neurodegenerative diseases such as Parkinson's disease, Alzheimer's disease (AD), and Huntington disease, but also, most importantly, to remove the source of the problem. Neural stem cells are capable of improving cognitive function in preclinical rodent models of $\mathrm{AD}$. It is clinically derived relevant human iPSCs from skin punch biopsies to develop a neural stem cell-based approach for treating AD. Neuronal degeneration in Parkinson's disease (PD) is focal, and dopaminergic neurons can be efficiently generated from hESCs. PD is an ideal disease for iPSC-based cell therapy.

Teeth represent a very challenging material for regenerative medicine. there is a chance for stem cells to become more widely used than synthetic materials. Teeth have a large advantage of being the most natural and non-invasive source of stem cells. For now, without the use of stem cells, the most common periodontological treatments are either growth factors, grafts, or surgery. For example, there are stem cells in periodontal ligament, which are capable of differentiating into osteoblasts or cementoblasts, and their functions were also assessed in neural cells. Tissue engineering is a successful method for treating periodontal diseases. Stem cells of the root apical areas are able to recreate periodontal ligament. One of the possible methods of tissue engineering in periodontology is gene therapy performed using adenoviruses-containing growth factors. As a result of animal studies, dentin regeneration is an effective process that results in the formation of dentin bridges. Enamel is more difficult to regenerate than dentin. After the differentiation of ameloblastoma cells into the enamel, the former is destroyed, and reparation is impossible. Medical studies have succeeded in differentiating bone marrow stem cells into ameloblastoma. Healthy dental tissue has a high amount of regular stem cells, although this number is reduced when tissue is either traumatized or inflamed. There are several dental stem cell groups that can be isolated.

The European Medicines Agency and the Food and Drug Administration have set Good Manufacturing Practice (GMP) guidelines for safe and appropriate stem cell transplantation. NutriStem with LN-511, TeSR2 with human recombinant laminin (LN-511), and RegES with human foreskin fibroblasts (HFFs) are commonly used xeno-free culture systems. There are many organizations and international initiatives, such as the National Stem Cell Bank, that provide stem cell lines for treatment or medical research.

After several decades of experiments, stem cell therapy is becoming a magnificent game changer for medicine. With 


\section{International Journal of Engineering Applied Sciences and Technology, 2021 Vol. 6, Issue 5, ISSN No. 2455-2143, Pages 178-188 \\ Published Online September 2021 in IJEAST (http://www.ijeast.com)}

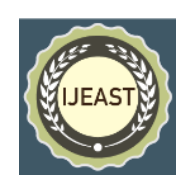

each experiment, the capabilities of stem cells are growing, although there are still many obstacles to overcome.

With stem cell therapy and all its regenerative benefits, we are better able to prolong human life than at any time in history.I

\section{ETHICAL ISSUES}

Human stem cell (hSC) research also raises sharp ethical and political controversies. The derivation of pluripotent stem cell lines from oocytes and embryos is fraught with disputes regarding the onset of human personhood and human reproduction. Several other methods of deriving stem cells raise fewer ethical concerns. The reprogramming of somatic cells to produce induced pluripotent stem cells (iPS cells) avoids the ethical problems specific to embryonic stem cells. With any hSC research, however, there are difficult dilemmas, including consent to donate materials for hSC research, early clinical trials of hSC therapies, and oversight of hSC research.

\section{New embryonic stem cell lines from frozen embryos}

Women and couples who undergo infertility treatment often have frozen embryos remaining after they complete their infertility treatment. The disposition of these frozen embryos is often a difficult decision for them to make. Some choose to donate these remaining embryos to research rather than giving them to another couple for reproductive purposes or destroying them. Several ethical concerns come into play when a frozen embryo is donated, including informed consent from the woman or couple donating the embryo, consent from gamete donors involved in the creation of the embryo, and the confidentiality of donor information.

\section{$A$. Informed consent for donation of materials for stem cell research.}

Since the Nuremburg Code, informed consent has been regarded as a basic requirement for research with human subjects. Consent is particularly important in research with human embryos. Obtaining informed consent for potential future uses of the donated embryo respects this diversity of views. Additionally, people commonly place special emotional and moral significance on their reproductive materials, compared with other tissues.

\section{$B$. Waiver of consent.}

In the United States, federal regulations on research permit a waiver of informed consent for the research use of deidentified biological materials that cannot be linked to donors. Thus, logistically it would be possible to carry out embryo and stem cell research on deidentified materials without consent. If infertility patients have frozen embryos remaining after they complete treatment, they are routinely contacted by the IVF program to decide whether they want to continue to store the embryos (and to pay freezer storage fees), to donate them to another infertile woman or couple, or to discard them. If a patient chooses to discard the embryos, it would be possible to instead remove identifiers and use them for research.

The ethical justifications for allowing deidentified biological materials to be used for research without consent do not always hold for embryo research .

\section{$C$. Consent from gamete donors.}

Frozen embryos may be created with sperm or oocytes from donors who do not participate any further in assisted reproduction or childrearing. ART clinics can readily discuss donation for research with oocyte donors during visits for oocyte stim

ulation and retrieval. However, most ART clinics obtain donor sperm from sperm banks and generally have no direct contact with the donors. Furthermore, sperm is often donated anonymously to sperm banks, with strict confidentiality provisions.

\section{$D$. Confidentiality of donor information.}

Confidentiality must be carefully protected in embryo and hESC research because

breaches of confidentiality might subject donors to unwanted publicity or even harassment by opponents of hESC research (20). Although identifying information about donors must be retained in case of audits by the Food and Drug Administration as part of the approval process for new therapies, concerns about confidentiality may deter some donors from agreeing to be recontacted.

Ethical concerns about oocyte donation for research are particularly serious:

\section{A. Medical risks of oocyte retrieval.}

The medical risks of oocyte retrieval include ovarian hyperstimulation syndrome, bleeding, infection, and complications of anesthesia. Because severe hyperovulation syndrome may require hospitalization or surgery, women donating oocytes for research should be protected against the costs of complications of hormonal stimulation and oocyte retrieval. CIRM allows state stem cell grants to cover the cost of such insurance. The rationale for making research institutions responsible for treatment is that they are in a better position than individual researchers to identify insurance policies and would have an incentive to consider extending such coverage to other research injuries.

\section{$B$. Protecting the reproductive interests of women in infertility treatment. \\ If women in infertility treatment share oocytes with researchers - either their own oocytes or those from an oocyte donor-their prospect of reproductive success may be compromised because fewer oocytes are available for reproductive purposes. In this situation, the physician carrying out oocyte retrieval and infertility care should give priority to}




\section{International Journal of Engineering Applied Sciences and Technology, 2021 Vol. 6, Issue 5, ISSN No. 2455-2143, Pages 178-188 \\ Published Online September 2021 in IJEAST (http://www.ijeast.com)}

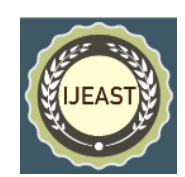

the reproductive needs of the patient in IVF. The highest quality oocytes should be used for reproductive purposes.

\section{Payment to oocyte donors.}

Many jurisdictions have conflicting policies about payment to oocyte donors. Reimbursement to oocyte donors for out-ofpocket expenses presents no ethical problems because donors gain no financial advantage from participating in research. On a pragmatic level, without such payment, it is very difficult to recruit oocyte donors for research.

Transplantation of cells derived from pluripotent stem cells offers the promise of effective new treatments. However, such transplantation also involves great uncertainty and the possibility of serious risks. Some stem cell therapies have been shown to be effective and safe, for example hematopoietic stem cell transplants for leukemia and epithelial stem cell-based treatments for burns and corneal disorders.

These clinical trials should follow ethical principles that guide all clinical research, including appropriate balance of risks and benefits and informed, voluntary consent:

\section{$A$. Risks and prospective benefits in stem cell clinical trials}

The risks of innovative stem cell-based interventions include tumor formation, immunological reactions, unexpected behavior of the cells, and unknown long-term health effects. Evidence of safety and proof of principle should be established through appropriate preclinical studies in relevant animal models or through human studies of similar cell-based interventions. Requirements for proof of principle and safety should be higher if cells have been manipulated extensively in vitro or have been derived from pluripotent stem cells.

\section{$B$. Informed consent in early stem cell clinical trials}

Several measures may enhance informed consent in early stem cell-based clinical trials.

First, researchers should describe the risks and prospective benefits in a realistic manner. Researchers need to communicate the distinction between the long-term hope for effective treatments and the uncertainty inherent in any phase I trial.

Second, investigators in hESC clinical trials should discuss a broader range of information with potential participants than in other clinical trials. The doctrine of informed consent requires researchers to discuss with potential participants information that is pertinent to their decision to volunteer for the clinical trial. Researchers in clinical trials of hESC transplantation should inform eligible participants that transplanted materials originated from human embryos.
Third, and most important, researchers should verify that participants have a realistic understanding of the clinical trial. The crucial ethical issue about informed consent is not what researchers disclose in consent forms or discussions, but rather what the participants in clinical trials understand.

HSC research offers exciting opportunities for scientific advances and new therapies, but also raises some complex ethical and policy issues. These issues need to be discussed along with scientific challenges to ensure that stem cell research is carried out in an ethically appropriate manner.I

\section{CONCLUSION}

From this study we can conclude that ongoing research on stem cell therapies gives hope to patients who would normally not receive treatment to cure their disease. Stem cells have a bright future for the therapeutic world by promising stem cell therapy. We hope to see new horizon of therapeutics in the form of bone marrow transplant, skin replacement, organ development, and replacement of lost tissue such as hairs, tooth, retina and cochlear cells.After several decades of experiments, stem cell therapy is becoming a magnificent game changer for medicine. With each experiment, the capabilities of stem cells are growing, although there are still many obstacles to overcome. Regardless, the influence of stem cells in regenerative medicine and transplantology is immense. Currently, untreatable neurodegenerative diseases have the possibility of becoming treatable with stem cell therapy. Induced pluripotency enables the use of a patient's own cells. Tissue banks are becoming increasingly popular, as they gather cells that are the source of regenerative medicine in a struggle against present and future diseases. With stem cell therapy and all its regenerative benefits, we are better able to prolong human life than at any time in history. The discovery of non-hematopoietic precursor cells in the bone marrow of human adults has shown that this organ harbors stem cells that can generate not only blood cells but also other tissues. Very recently, cells of various tissue types have been generated from bone marrow-derived progenitor cells. Mesenchymal stem cells from the bone marrow can be driven to differentiate into osteoblasts, chondrocytes, fibroblasts and adipocytes. Bone marrow-derived cells can adopt the fate of endothelial cells, hepatocytes, neural cells, and other cells in animal transplant models. The bone marrow-derived endothelial cells can contribute to maintenance angiogenesis, that is, the replacement of endothelial cells that are lost from the blood vessel wall. The development of treatment methods has evoked great expectations and have offered much hope by promising to greatly extend the numbers and range of patients who could benefit from transplants, and to provide cell replacement therapy to treat debilitating diseases such as diabetes, Parkinson's, Alzheimer's and Huntington's disease. Though human stem cell (hSC) research also raises sharp ethical and political controversies but scientists are fighting against all those issues. Stem cell research leading to 


\section{International Journal of Engineering Applied Sciences and Technology, 2021 \\ Vol. 6, Issue 5, ISSN No. 2455-2143, Pages 178-188 \\ Published Online September 2021 in IJEAST (http://www.ijeast.com)}

prospective therapies in reparative medicine has the potential to affect the lives of millions of people around the world for the better good reason.

\section{REFERENCE}

1. Preleukemic Hematopoietic Stem Cells in Human Acute Myeloid Leukemia

by M. Ryan Corces, Howard Y. Chang and Ravindra Majeti. doi: 10.3389/fonc.2017.00263

2. Pre-leukemic evolution of hematopoietic stem cells: the importance of early mutations in leukemogenesis

by MR Corces-Zimmerman 1 and R Majeti.

doi:10.1038/leu.2014.211

3. Leukaemia stem cells and the evolution of cancer-stem-cell research

by Brian J. P. Huntly and D. Gary Gilliland

doi: $10.1038 /$ nrc1592

4. Ethical Issues in Stem Cell Research

by Bernard Lo and Lindsay Parham

doi: 10.1210/er.2008-0031

5. History and perspective of stem cell research by Ariff Bongso and Mark Richards doi:10.1016/j.bpobgyn.2004.09.002

6. Stem Cell Research in Stroke, How Far From the Clinic? by Olle Lindvall, MD, PhD; Zaal Kokaia, PhD doi: 10.1161/STROKEAHA.110.599654

7. Recent advances in stem cell neurobiology

by $\mathrm{T}$ Ostenfeld, CN Svendsen

doi: 10.1007/978-3-7091-0641-9_1

8. Role of Stem Cells in Treatment of Neurological DISORDER

by Ashfaq UI Hassan, Ghulam Hassan and Zahida Rasool. PMCID: PMC3068820

9. The Ethics of Human Stem Cell Research

by Gene H. Outka,

https://doi.org/10.1186/s40779-018-0154-9

10. Application of stem cells in tissue engineering for defense medicine

by Chinedu Cletus Ude1, Azizi Miskon, Ruszymah Bt $\mathrm{Hj}$ Idrus and Muhamad Bin Abu Bakar

https://doi.org/10.1186/s40779-018-0154-9
11. Stem cells: past, present, and future by Wojciech Zakrzewski1, Maciej Dobrzyński, Maria Szymonowicz and Zbigniew Rybak https://doi.org/10.1186/s13287-019-1165-5

12. Stem Cells in Wound Healing: The Future of Regenerative Medicine?

A Mini-Review by Dominik Duscher, Janos Barrera, Victor W. Wong, Zeshaan N. Maan Alexander J. Whittam, Michael Januszyk, Geoffrey C. Gurtner

DOI: $10.1159 / 000381877$

13. The future for stem cell research by Robin Lovell-Badge

\begin{tabular}{l|ll|lll|} 
NATURE & VOL $414 \quad 1$ & NOVEMBER & 2001
\end{tabular} www.nature.com

14. Mesenchymal Stem Cells

by Arnold I. Caplan, J Orthop Res, Vol. 9, No. 5, 1991

15. Stem cells: Concepts and prospects

by SAVNEET KAUR1 and C C KARTHA

https://www.ias.ac.in/public/Resources/Other_Publications/Ov erview/Current_Trends/437-452.pdf

16. Preleukemic Hematopoietic Stem Cells in Human Acute Myeloid Leukemia

by M. Ryan Corces , Howard Y. Chang and Ravindra Majeti https://doi.org/10.3389/fonc.2017.00263

17. Hematopoietic stem cells: concepts, definitions, and the new reality

by Connie J. Eaves ,BLOOD, 23 APRIL 2015 x VOLUME 125, NUMBER 17

18. Hematopoietic stem cells

by E. Gunsilius*, G. Gastl, A.L. Petzer Biomed Pharmacother $2001 ; 55: 186-94$

(C) 2001 Éditions scientifiques et médicales Elsevier SAS

19. https://www.ahajournals.org/journal/str

20. THE BIOLOGY OF HEMATOPOIETIC STEM CELLS by Sean J. Morrison, Nobuko Uchida1, and Irving L. Weissman Annu. Rev. Cell Dev. Biol. 1995.11:35-71.

21. Differentiation and Proliferation of Hematopoietic Stem Cells

by Makio Ogawa Blood. Vol81, No 11 (June 1), 1993: pp 2844-2853

22. Stem Cell: Basics, Classification and Applications by K. Kalra* and P.C. Tomar AJPCT[2][7][2014]919-930 